\title{
Consideraciones generales para la elaboración de un atlas de almacenamiento geológico de $\mathrm{CO}_{2}$ del Perú
}

\author{
Jorge Humberto Paredes-Ángeles ${ }^{1}$
}

Forma de citar: Paredes-Ángeles, J.H. (2020). Consideraciones generales para la elaboración de un atlas de almacenamiento geológico de $\mathrm{CO}_{2}$ del Perú. Boletín de Geología, 42(3), 243-260. https://doi.org/10.18273/revbol.v42n3-2020011

\begin{abstract}
Resumen
La captura y el secuestro o almacenamiento geológico de $\mathrm{CO}_{2}$ es la mejor alternativa para reducir el exceso de emisiones de $\mathrm{CO}_{2}$ antropogénico al medio ambiente, tanto por consideraciones técnicas como económicas. Los volúmenes de almacenamiento de $\mathrm{CO}_{2}$ y los tiempos de residencia prolongados en los depósitos geológicos lo hacen particularmente interesante. Aunque no se ha probado a gran escala, los diferentes proyectos piloto realizados y los que están en ejecución en varias partes de Europa y Norteamérica muestran resultados muy alentadores.

En este trabajo se desarrollan los principios más importantes que hay que tomar en cuenta al elaborar un atlas de almacenamiento geológico de $\mathrm{CO}_{2}$. Se hace una revisión de los fundamentos teórico-prácticos para el secuestro geológico de $\mathrm{CO}_{2}$, también se desarrollan los tipos y mecanismos de retención del dióxido de carbono en las diferentes formaciones geológicas, luego se revisan los principales criterios de selección de los emplazamientos y los indicadores de idoneidad de los sitios para el secuestro de dióxido de carbono. Se presenta un sistema de clasificación de los sitios en función del conocimiento geológico de la formación seleccionada que es una adaptación de la experiencia geológica en la exploración de petróleo y gas. Se proponen tentativamente las áreas geográficas a investigar e información geológica a recabar y procesar en función a los potenciales almacenes existentes y también se expone la metodología para la estimación de los recursos de almacenamiento o secuestro geológico de dióxido de carbono en función de las formaciones que podrían ser encontradas.
\end{abstract}

Palabras clave: Secuestro de $\mathrm{CO}_{2}$; Almacenes geológicos de $\mathrm{CO}_{2}$; Formaciones geológicas almacén; Atlas de almacenamiento de $\mathrm{CO}_{2}$ en Perú.

\section{General considerations for the development of the Peruvian atlas of $\mathrm{CO}_{2}$ geological storage}

\begin{abstract}
$\mathrm{CO}_{2}$ capture and sequestration or geological storage is the best alternative for reducing excess anthropogenic $\mathrm{CO}_{2}$ emissions into the environment, both for technical and economic considerations. The volumes of $\mathrm{CO}_{2}$ and the prolonged residence times in the geological deposits make it especially interesting. Although it has not been tested on a large scale, the different pilot projects carried out and those underway in various parts of the world have shown very encouraging results.

This work develops the most important principles to be taken into account when preparing a geological atlas for storage of $\mathrm{CO}_{2}$. The theoretical-practical foundations for the geological sequestration of $\mathrm{CO}_{2}$ are reviewed, the types and mechanisms of carbon dioxide sequestration in the different geological formations are also developed, then is reviewed the main criteria for the selection of sites and the indicators of suitability for the sequestration of carbon dioxide. It presents a system of classification of sites according to the geological knowledge of the selected formation, which is an adaptation of geological experience in oil and gas exploration. It tentatively proposes geographical areas to investigate and collect geological information based on existing potential storage sites and it also exposes a methodology for estimating the storage capacity for geological sequestration of carbon dioxide depending on the formations that could be found.
\end{abstract}

Keywords: $\mathrm{CO}_{2}$ sequestration; $\mathrm{CO}_{2}$ geological stores; Geological formations for storage; Atlas of $\mathrm{CO}_{2}$ storage in Peru.

${ }^{1}$ Universidad Nacional de Ingeniería, Lima, Perú. jhparedes@gmail.com 


\section{Introducción}

El Perú (ver Figura 1), no es un importante consumidor de energía, pero de la evolución de su matriz eléctrica en los últimos años; es posible apreciar que la quema de combustibles fósiles (carbón, petróleo y gas, por lo tanto, la generación de $\mathrm{CO}_{2}$ ) se ha casi duplicado de 2006 al 2016 (ver Figura 2), y ahora es la principal fuente generadora de electricidad en el Perú; lo que convierte a este país también en parte del problema de la generación de gases de efecto invernadero.

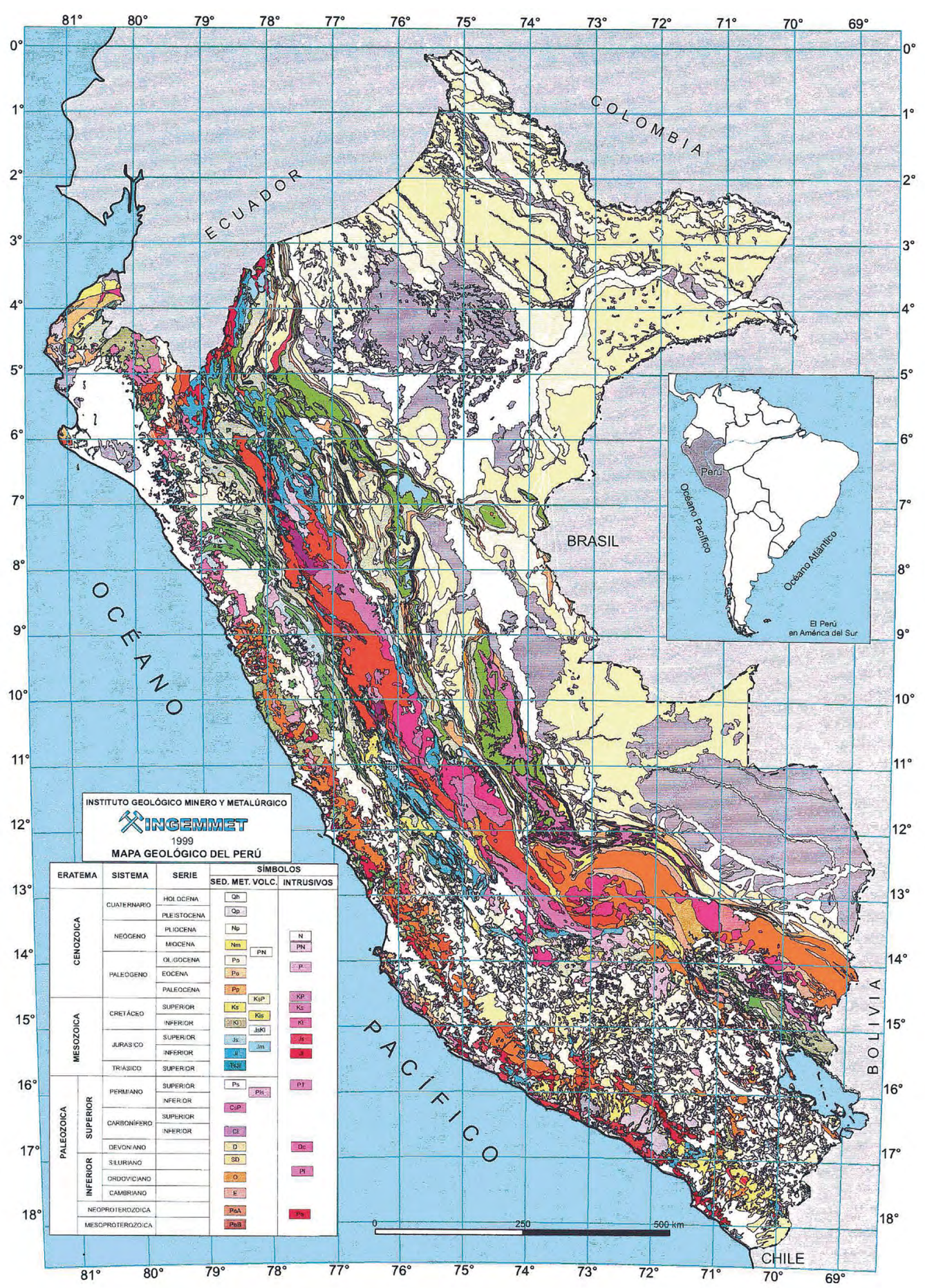

Figura 1. Mapa de ubicación del área de estudio/mapa geológico del Perú (modificado de INGEMMET, 1999). 


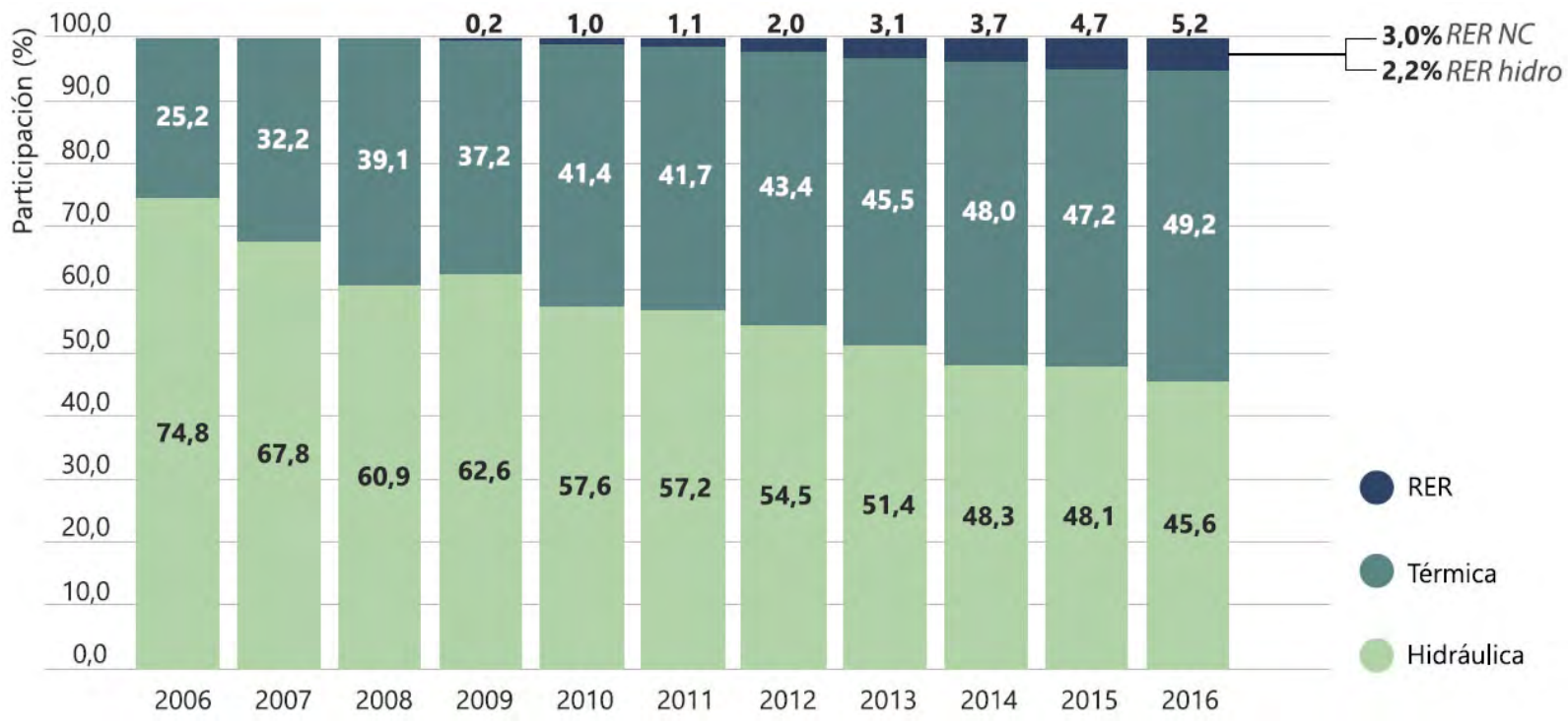

Figura 2. Evolución de la Matriz energética del Perú 2016-2016 (Dirección General de Electricidad, 2016).

El acuerdo de París de diciembre 2015 se traza como objetivo mantener el incremento de la temperatura global muy por debajo de $\operatorname{los} 2^{\circ} \mathrm{C}$, respecto a los niveles pre industriales (Naciones Unidas UNFCC, 2015). Además, proseguir los esfuerzos para limitar ese aumento a $1,5^{\circ} \mathrm{C}$ y evitar así efectos catastróficos para los países más vulnerables (Naciones Unidas UNFCC, 2015). Y respecto a las emisiones de gases contaminantes dice: "lograr el equilibrio entre los gases emitidos y los que pueden ser absorbidos en la segunda mitad del siglo, en otras palabras, ser carbono neutral" (Naciones Unidas UNFCC, 2015); en esta dirección algunos países de la comunidad europea, américa del norte y Australia han trabajado ya sus atlas de almacenamiento geológico de $\mathrm{CO}_{2}$ (ver Tabla 1) y desarrollan una serie de otras actividades relacionadas. En el Perú aún no ha sido tomada ninguna medida ni gubernamental ni privada para dimensionar el potencial de almacenamiento geológico de $\mathrm{CO}_{2}$.

Los atlas a diferencia de los Sistemas de Información Geográficos (SIG), son colecciones de mapas temáticos específicos, estáticos y por lo tanto quedan fijados en un documento, que puede ser alguna variante de reporte final. Los SIG son dinámicos y requieren constante actualización o al menos su mantenimiento en sitios web especializados que requieren conocimientos y asignación de presupuesto determinados.

Tabla 1. Cuadro comparativo de Atlas Nacionales de Captura y Almacenamiento Geológico de $\mathrm{CO}_{2}$.

\begin{tabular}{lll}
\hline \multicolumn{1}{c}{ País } & \multicolumn{1}{c}{ Título del trabajo } & \multicolumn{1}{c}{ Principales características } \\
\hline & & Ejecutor: $\mathrm{CO}_{2}$ Geological Storage Solutions (CGSS) \\
& An assessment of Queensland's & Idioma: inglés \\
Australia (Queens- & $\mathrm{CO}_{2}$ geological storage prospectiv- & Ediciones: 01, Año: 2011 \\
land) & $\begin{array}{l}\text { ity - the Queensland } \mathrm{CO}_{2} \text { Geo- } \\
\text { logical Storage Atlas (Bradshaw }\end{array}$ & Tópicos desarrollados: \\
& et al., 2011) & - Cuencas de alto potencial (mapas) \\
& - Cuencas de bajo potencial e inadecuadas (Mapas) \\
\hline
\end{tabular}

\begin{tabular}{|c|c|c|}
\hline \multirow{7}{*}{ Brasil } & \multirow{7}{*}{$\begin{array}{l}\text { Atlas Brasileiro de Captura e } \\
\text { Armazenamento Geológico de } \mathrm{CO}_{2} \\
\text { (Medina-Ketzer et al., 2016) }\end{array}$} & $\begin{array}{l}\text { Ejecutor: Centro de Excelência em Pesquisa e Inovação em Petróleo, Recursos Mine- } \\
\text { rais e Armazenamento de Carbono (CEPAC) [Petrobras y Pontificia Universidad Cató- } \\
\text { lica de Rio Grande do Sul (PUCRS)] }\end{array}$ \\
\hline & & Idioma: Postugués e inglés \\
\hline & & Ediciones: 01, Año: 2016 \\
\hline & & Tópicos desarrollados: \\
\hline & & - Aspectos generales (marco general) \\
\hline & & - Situación de Brasil (Evaluación fuentes, captura, transporte y almacenamiento) \\
\hline & & - Potencial en el Brasil (Mapas) \\
\hline
\end{tabular}


Continuación Tabla 1.

\begin{tabular}{|c|c|c|}
\hline País & Título del trabajo & Principales características \\
\hline $\begin{array}{l}\text { Estados Unidos (y } \\
\text { parte de Canadá) }\end{array}$ & $\begin{array}{l}\text { Carbon Storage Atlas (5th Edition) } \\
\text { (DOE \& NETL, 2015) }\end{array}$ & $\begin{array}{l}\text { Ejecutor: Department of Energy's (DOE) National Energy Technology Laboratory } \\
\text { (NETL) } \\
\text { Idioma: inglés } \\
\text { Ediciones: 05, Última Año: } 2015 \\
\text { Tópicos desarrollados: } \\
\text { - Aspectos generales (marco general, teoría del almacenamiento, fuentes) } \\
\text { - Atlas (mapas) } \\
\text { - Proyectos de gran escala (Ejemplos descriptivos) } \\
\text { - Proyectos de pequeña escala (Ejemplos descriptivos) } \\
\text { - Resumen de las metodologías aplicadas }\end{array}$ \\
\hline México & $\begin{array}{l}\text { Atlas de Almacenamiento Geológi- } \\
\text { co de } \mathrm{CO}_{2} \text { México (Beltrán et al., } \\
\text { 2012) }\end{array}$ & $\begin{array}{l}\text { Ejecutor: Secretaría de Energía (SENER) Comisión Federal de Electrecidad (CFE) } \\
\text { Idioma: Castellano } \\
\text { Ediciones: 01, Año: } 2012 \\
\text { Tópicos desarrollados: } \\
\text { - Aspectos generales (marco general) } \\
\text { - Emisiones de } \mathrm{CO}_{2} \text { en México } \\
\text { - Almacenamiento geológico (Marco teórico) } \\
\text { - Capacidades de almacenamiento geológico de } \mathrm{CO}_{2} \text { en México (Incluye Mapas) }\end{array}$ \\
\hline Noruega & $\begin{array}{l}\mathrm{CO}_{2} \text { atlas for the Norwegian Conti- } \\
\text { nental Shelf (Norwegian Petroleum } \\
\text { Directorate, 2014) }\end{array}$ & $\begin{array}{l}\text { Ejecutor: Norwegian Petroleum Directorate } \\
\text { Idioma: inglés } \\
\text { Ediciones: Parciales, Año: 2011-2013 / Total, Año: } 2014 \\
\text { Tópicos desarrollados: } \\
\text { - Aspectos generales (marco general, teoría del almacenamiento, fuentes) } \\
\text { - Metodología } \\
\text { - Descripción Geológica } \\
\text { - Opciones de Almacenamiento } \\
\text { - Atlas del mar del Norte } \\
\text { - Atlas del mar de Noruega } \\
\text { - Atlas del mar de Barents } \\
\text { - Resumen de la capacidad de almacenamiento }\end{array}$ \\
\hline
\end{tabular}

\section{Marco teórico}

\section{Secuestro geológico del carbono}

La captura y almacenamiento o secuestro del dióxido de carbono es el proceso de captación y retención del $\mathrm{CO}_{2}$ evitando que se acumule en la atmósfera. Desde la academia se está investigando una serie de variantes para la captura y almacenamiento o secuestro de $\mathrm{CO}_{2}$, entre ellas el secuestro geológico del dióxido carbono. El secuestro o almacenamiento geológico se define como la colocación de $\mathrm{CO}_{2}$ en una formación geológica subterránea de tal manera que permanezca almacenado permanentemente (DOE - Laboratory, U.D., 2010; CO2CRC, 2012).

En el mundo se están estudiando varios tipos de formaciones geológicas para el secuestro o almacenamiento del dióxido de carbono; cada uno de ellos tiene sus particularidades especiales: (1) depósitos de petróleo y gas; (2) salmueras o formaciones salinas profundas; (3) mantos de carbón no explotable (4) esquistos ricos en orgánicos; y (5) formaciones de basalto; y más recientemente (6) formaciones de esquisto fracturado agotadas por hidrocarburos; (7) otras opciones incluyen evaporitas, pizarras bituminosas y cavidades subterráneas (Figura 3).

El proceso de secuestro geológico de dióxido de carbono incluye el monitoreo, la verificación, el registro y la evaluación de riesgos en el sitio de almacenamiento. Estas actividades de los diferentes entes encargados de la reducción de gases de efecto invernadero en el mundo se centran en el desarrollo y la implementación de tecnologías que pueden proporcionar un registro preciso del $\mathrm{CO}_{2}$ almacenado y un alto nivel de confianza en que el $\mathrm{CO}_{2}$ se mantendrá permanentemente almacenado.

Relacionado con el gradiente geotérmico y con la profundidad, el $\mathrm{CO}_{2}$ inyectado alcanza el estado supercrítico comportándose como un fluido denso (a presiones mayores que $7,38 \mathrm{MPa}$ y temperaturas mayores que $31,1^{\circ} \mathrm{C}$ ) (Figura 4). En esta condición, el $\mathrm{CO}_{2}$ adquiere una densidad similar a la del líquido, entre 600 y $800 \mathrm{~kg} / \mathrm{m}^{3}$, hecho por el que ocupa un menor volumen de poro, lo que permite un almacenamiento más eficiente (Figura 5). Si queremos asegurar el almacenamiento en estado supercrítico, la profundidad mínima establecida para un reservorio es de cerca de $800 \mathrm{~m}$, en la que se dan estas condiciones. 


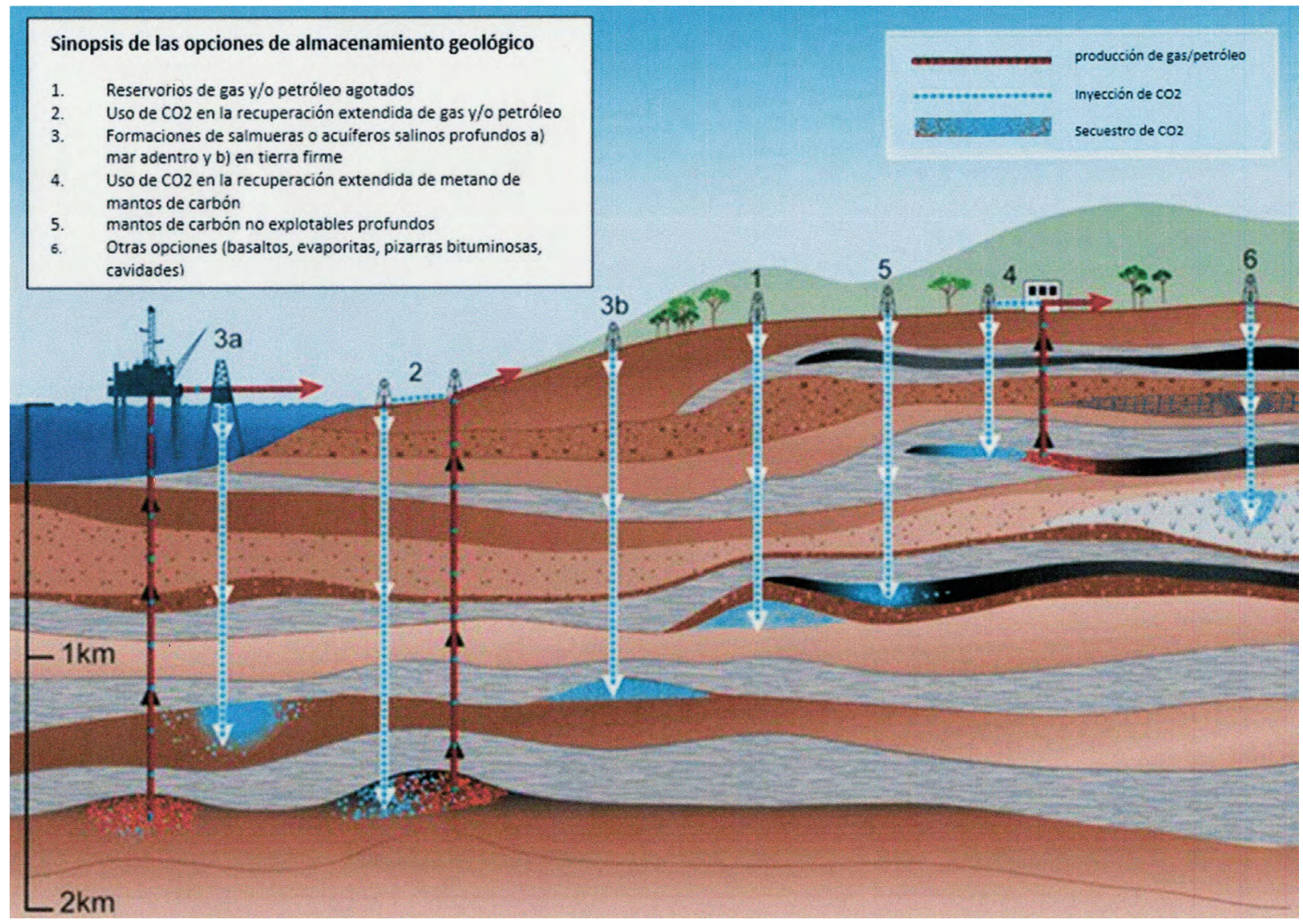

Figura 3. Sinopsis de las opciones de secuestro geológico de $\mathrm{CO}_{2}$ (modificado de PNUMA, 2005).

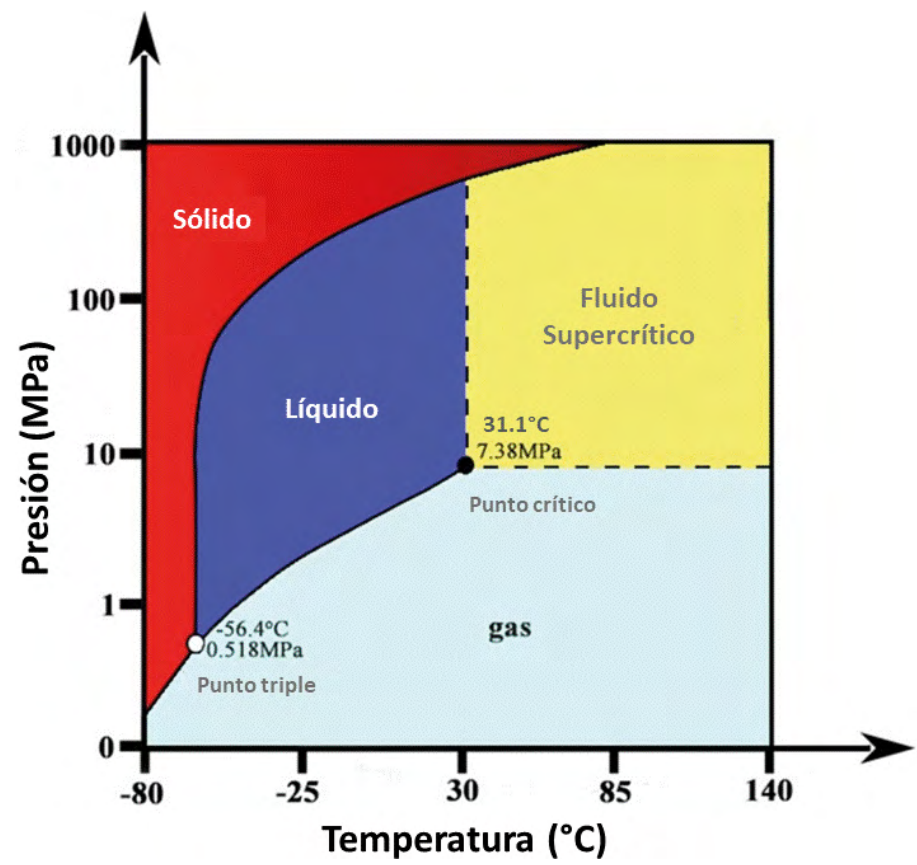

Figura 4. Diagrama de fases de $\mathrm{CO}_{2}$ (modificado de ChemicaLogic Corporation, 1999). 


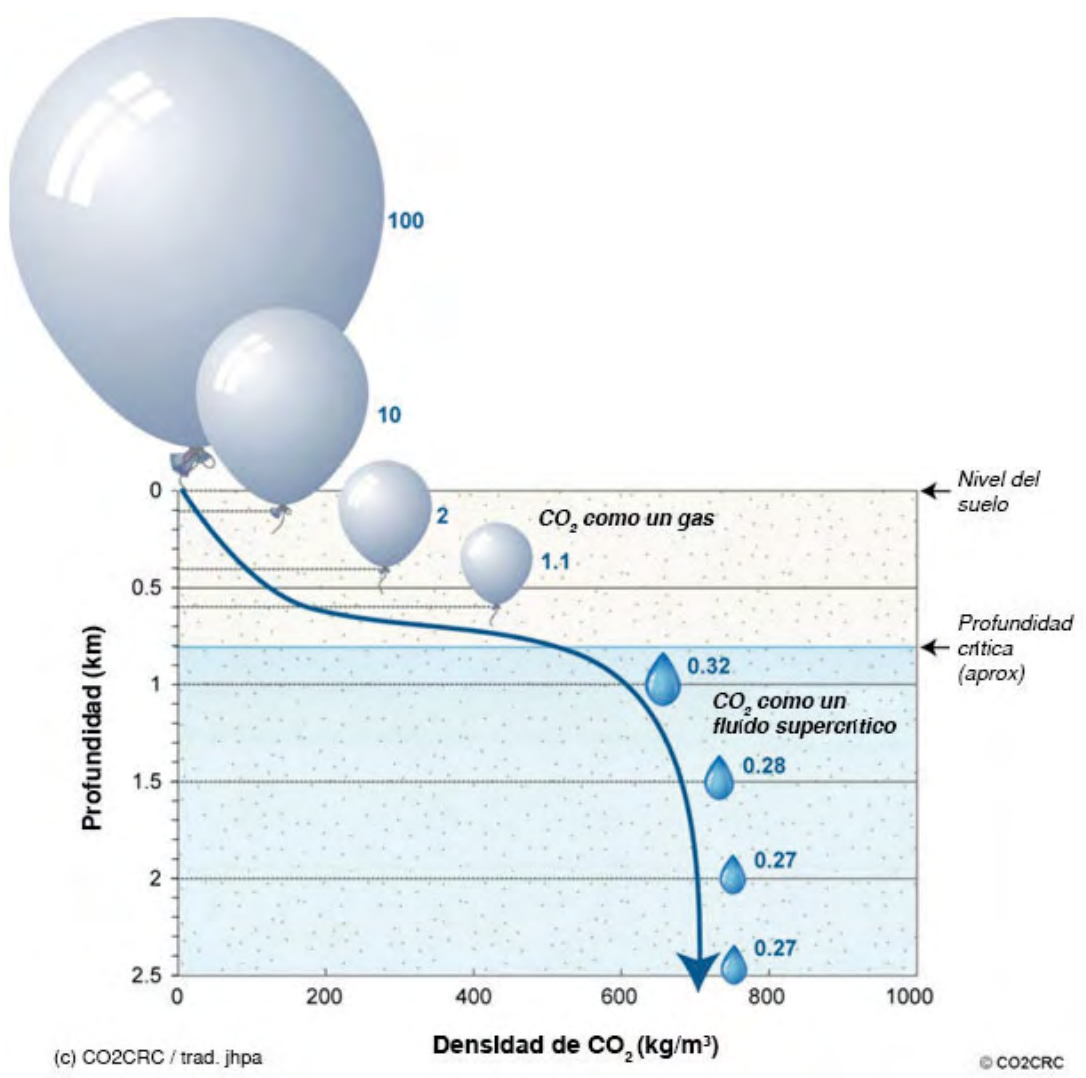

Figura 5. Diagrama de cambio de volumen por profundidad y temperatura (modificado de CO2CRC, 2012).

Cada una de las opciones de almacenamiento geológico tiene sus peculiaridades y rasgos distintivos que es necesario identificar y desarrollar. hidrodinámicos (residual y adsorción), como químicos (atrapamiento por dilución o formación mineral) (Leung et al., 2014), resumidos en la Tabla 2.

Los mecanismos de retención y/o captura son tanto físicos (trampa estructural o estratigráfica),

Tabla 2. Tipos y mecanismos de retención y/o captura del $\mathrm{CO}_{2}$ (modificado de Leung et al., 2014).

\begin{tabular}{|c|c|c|}
\hline $\begin{array}{l}\text { Mecanismo de retención y/o de } \\
\text { captura }\end{array}$ & Fase de captura de $\mathrm{CO}_{2}$ & Descripción del mecanismo \\
\hline $\begin{array}{l}\text { Hidrodinámica; captura de } \\
\text { movilidad }\end{array}$ & Fase supercrítica & $\begin{array}{l}\mathrm{El} \mathrm{CO} \mathrm{CO}_{2} \text { no disuelto queda atrapado por la presión que ejerce el } \\
\text { agua de la formación y el sello. }\end{array}$ \\
\hline Captura estructural o estratigráfica & Fase supercrítica & $\begin{array}{l}\mathrm{E} \mathrm{CO}_{2} \text { inyectado, se inmoviliza en trampas estructurales o } \\
\text { estratigráficas, como el petróleo o gas natural. }\end{array}$ \\
\hline Residual; captura capilar & Fase supercrítica & $\begin{array}{l}\mathrm{El} \mathrm{CO}_{2} \text { se eleva a través de la roca saturada de agua y desplaza el } \\
\text { agua del espacio poroso por acción de las fuerzas capilares; todo } \\
\text { el volumen de la roca retiene una saturación residual de } \mathrm{CO}_{2} \text {. }\end{array}$ \\
\hline $\begin{array}{l}\text { Solubilidad; captura por } \\
\text { disolución }\end{array}$ & Fase líquida disuelta & $\begin{array}{l}\mathrm{El} \mathrm{CO}_{2} \text { se disuelve en el agua de salmuera de formación; es un } \\
\text { muy importante mecanismo de captura. }\end{array}$ \\
\hline $\begin{array}{l}\text { Mineral, mineralización; o captura } \\
\text { química }\end{array}$ & Fase sólida de reacción & $\begin{array}{l}\mathrm{El} \mathrm{CO} \text { disuelto reacciona con } \mathrm{Ca}, \mathrm{Fe} \text { o } \mathrm{Mg} \text { para formar } \\
\text { carbonatos precipitados; no está sujeto a fugas. }\end{array}$ \\
\hline Adsorción, captura por adsorción & Fase gaseosa/ supercrítica & $\begin{array}{l}\mathrm{El} \mathrm{CO}_{2} \text { queda adherido a las paredes internas de los poros del } \\
\text { carbón }\end{array}$ \\
\hline
\end{tabular}




\section{Criterios de selección de los emplazamientos}

Considerando que los almacenes geológicos por su naturaleza son variados, en general rocas sedimentarias clásticas, mantos de carbón, rocas magmáticas básicas vesiculares, los criterios de selección también son variados.

Estos criterios incluyen los siguientes, pero no se limitan a: (1) condiciones de presión y temperatura; (2) aislamiento de aguas subterráneas potables poco profundas, otros estratos, suelos y atmósfera; y (3) presión de entrada de la capa sello (Bachu, 2008).
Los principales indicadores geológicos para establecer la idoneidad del sitio de almacenamiento para rocas sedimentarias clásticas son la porosidad y la permeabilidad, que disminuyen con el aumento de la profundidad.

Basado en este indicador en la Tabla 3 se evidencia que, la región óptima para el secuestro/almacenamiento geológico de $\mathrm{CO}_{2}$ está entre los 800 y $2500 \mathrm{~m}$ de profundidad.

Tabla 3. Indicadores geológicos clave para establecer la idoneidad del sitio de almacenamiento (modificado de Chadwick et al., 2008).

\begin{tabular}{lll}
\hline \multicolumn{1}{c}{ Propiedades del reservorio } & \multicolumn{1}{c}{ Indicadores positivos } & \multicolumn{1}{c}{ Indicadores de cautela } \\
\hline Profundidad & $>800 \mathrm{~m},<2500 \mathrm{~m}$ & $<800 \mathrm{~m},>2500 \mathrm{~m}$ \\
Espesor del reservorio & $>50 \mathrm{~m}$ & $<20 \mathrm{~m}$ \\
Porosidad & $>20 \%$ & $<10 \%$ \\
Salinidad & $>100 \mathrm{gL}^{-1}$ & $<30 \mathrm{gL}^{-1}$ \\
& Capacidad efectiva estimada mucho & Capacidad efectiva estimada similar al \\
Capacidad & mayor que el monto total de $\mathrm{CO}_{2}$ a ser & monto total de $\mathrm{CO}_{2}$ a ser inyectado \\
& inyectado & \\
\hline \multicolumn{1}{c}{ Propiedades del sello } & & \\
\hline \multirow{2}{*}{ Continuidad lateral } & Estratigráficamente uniforme, con & Variaciones laterales, fallas medianas o \\
Espesor / potencia & pequeñas o sin fallas & grandes \\
\hline
\end{tabular}

\section{Caracterización de sitios para almacenamiento geológico}

Este es un proceso semejante a los métodos que se emplean en la industria petrolera para madurar un proyecto, a través de una secuencia progresiva de clases de recursos y subclases de estado de desarrollo hasta que madure y comience a producir hidrocarburos. Un sistema de clasificación geológica de secuestro o almacenamiento de $\mathrm{CO}_{2}$ se considera debería seguir los mismos procesos desarrollados por el negocio petrolero en una progresión ascendente basada en análisis realizados para reducir el riesgo de desarrollo del proyecto (Tabla 4). El marco propuesto por el Departamento de Energía de los Estados Unidos (U.S. Department of Energy's DOE) contiene tres fases distintas de evaluación (fase de exploración, fase de caracterización del sitio y fase de implementación) que corresponden a cada clase de recurso y se subdividen en subclases de proyecto.

La información para el secuestro geológico de dióxido de carbono en nuestra propuesta de atlas se desarrolla para proporcionar una visión general de alto nivel de almacenamiento geológico de $\mathrm{CO}_{2}$ en el territorio peruano. Las extensiones superficiales de las formaciones geológicas y las estimaciones de recursos de almacenamiento de $\mathrm{CO}_{2}$ que se presenten deben ser considerados solamente como una evaluación inicial del potencial de almacenamiento geológico. El atlas en construcción proporcionará información básica sobre un sitio potencial antes de una evaluación de la fase de exploración, por lo tanto, debe tomarse como una primera aproximación. La maduración del sitio (Figura 6), corresponderá a futuros trabajos que se ejecuten progresivamente durante la exploración. 
Tabla 4. Comparación de la clasificación de la industria petrolera (Society of Petroleum Engineers, 2018) con la propuesta de clasificación de secuestro geológico de $\mathrm{CO}_{2}$ (Frailey y Finley, 2009).

\begin{tabular}{|c|c|c|c|}
\hline Industria petrolera & \multicolumn{2}{|c|}{$\begin{array}{l}\text { Almacenamiento geológico de } \\
\mathrm{CO}_{2}\end{array}$} & \\
\hline Reservas & \multirow{4}{*}{ 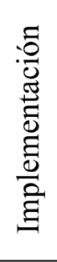 } & Capacidad & \\
\hline En producción & & Inyección activa & \\
\hline $\begin{array}{l}\text { Aprobado para } \\
\text { desarrollo }\end{array}$ & & Aprobado para el desarrollo & \\
\hline $\begin{array}{l}\text { Justificados para } \\
\text { desarrollo }\end{array}$ & & $\begin{array}{l}\text { Justificados para el } \\
\text { desarrollo }\end{array}$ & \\
\hline $\begin{array}{c}\text { Recursos } \\
\text { Contingentes }\end{array}$ & \multirow{4}{*}{ 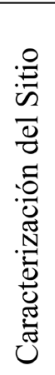 } & $\begin{array}{c}\text { Recursos de } \\
\text { Almacenamiento } \\
\text { Contingentes }\end{array}$ & \\
\hline Pendiente de desarrollo & & Desarrollo pendiente & \\
\hline $\begin{array}{l}\text { Desarrollo sin aclarar o } \\
\text { en espera }\end{array}$ & & $\begin{array}{l}\text { Desarrollo sin aclarar o en } \\
\text { espera }\end{array}$ & \\
\hline Desarrollo No Viable & & Desarrollo No Viable & \\
\hline $\begin{array}{c}\text { Recursos } \\
\text { prospectivos }\end{array}$ & \multirow{4}{*}{$\frac{: \frac{0}{0}}{\frac{\pi}{0}}$} & $\begin{array}{c}\text { Recursos de } \\
\text { almacenamiento } \\
\text { prospectivos }\end{array}$ & \\
\hline Prospecto & & Sitio(s) calificado(s) & \\
\hline Lead & & Áreas seleccionadas & \\
\hline \multirow[t]{6}{*}{ Play } & & Sub-Regiones tamizadas & \\
\hline & \multirow{5}{*}{ 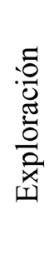 } & \multicolumn{2}{|c|}{ Recursos de almacenamiento prospectivos } \\
\hline & & Subclase de proyecto & Proceso de evaluación \\
\hline & & Sitio(s) calificado(s) & Caracterización inicial \\
\hline & & Áreas seleccionadas & Selección del sitio \\
\hline & & Sub-Regiones tamizadas & Evaluación del sitio \\
\hline
\end{tabular}

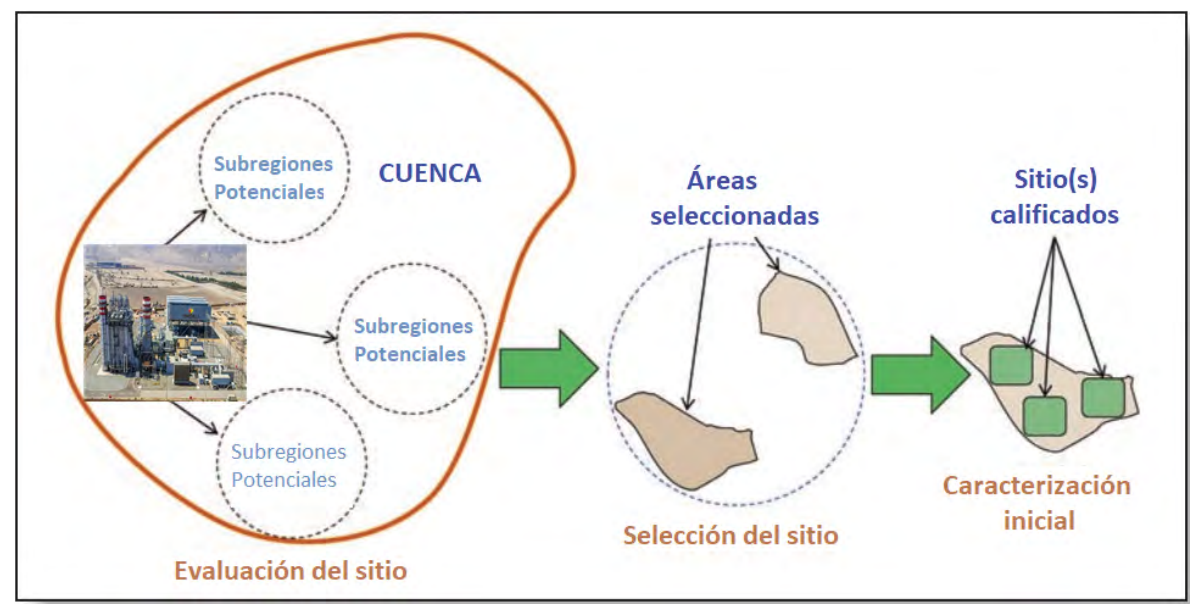

Figura 6. Representación gráfica de la maduración del sitio del proyecto a través de las diferentes fases de exploración. 


\section{Principales cuencas sedimentarias en el Perú}

Se han establecido 18 cuencas sedimentarias con posibilidades de exploración por hidrocarburos y de potencial almacenamiento de $\mathrm{CO}_{2}$, ocho de ellas están en o parcialmente costa-afuera (lo que se conoce como offshore) las demás se emplazan en la parte continental (Figura 7).
La importancia para los fines de estimación de los potenciales almacenes geológicos es muy grande, ya que en estas cuencas se sitúan los más importantes depósitos de hidrocarburos (petróleo, gas), así como también las formaciones que alojan aguas salinas.

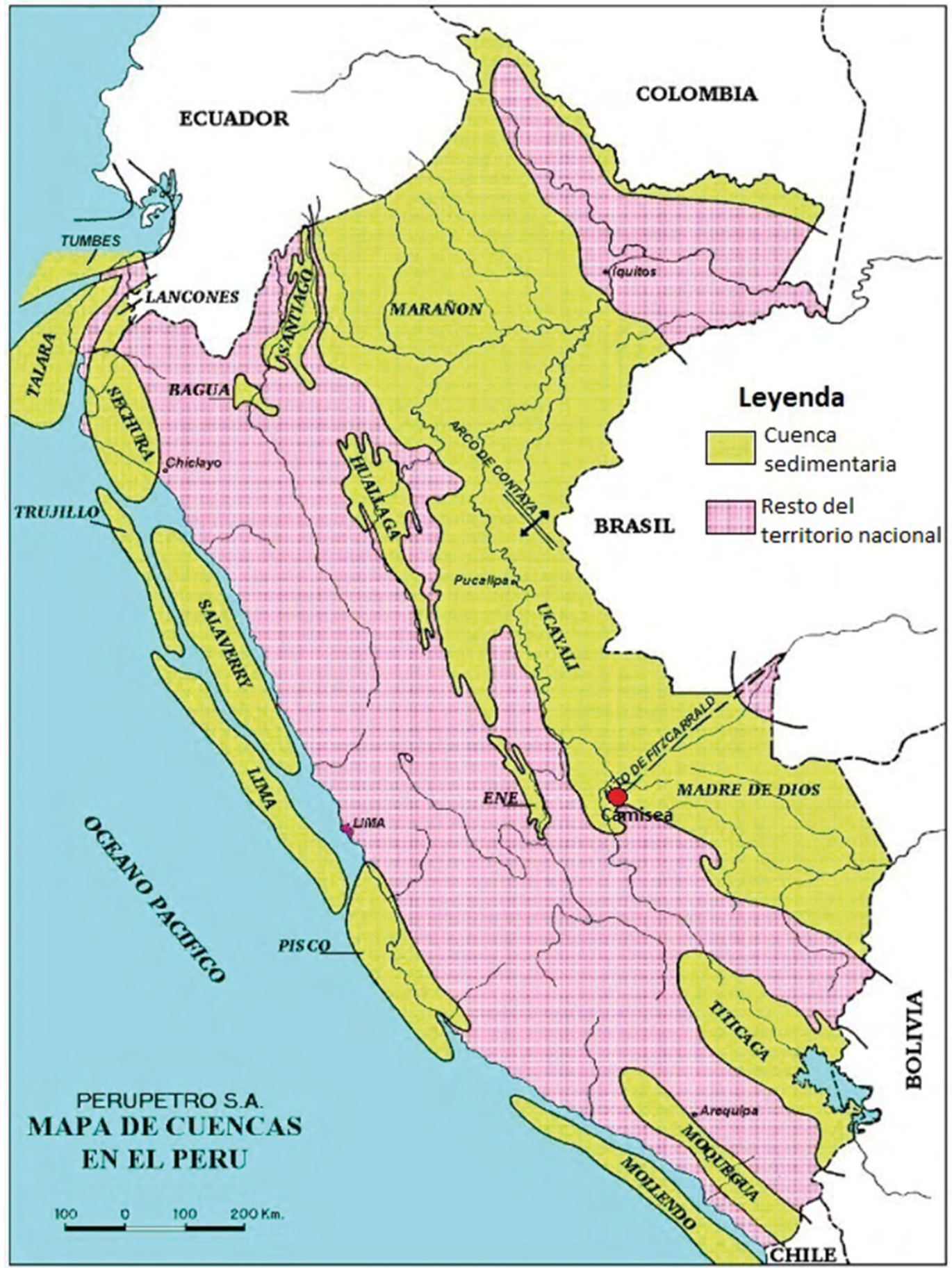

Figura 7. Cuencas sedimentarias del Perú (modificado de MINEM, 2017). 
En las cuencas del norte Tumbes-Progreso y Talara, desde inicios del siglo pasado se ha tenido producción comercial de petróleo, en especial en la Cuenca Talara que ha sido intensamente explotada y que ha acumulado una producción de alrededor de 1,500 millones de barriles de petróleo.

Las demás cuencas sedimentarias emplazadas en el zócalo continental no han sido exploradas cabalmente, algunas con levantamientos sísmicos y perforación de algunos pozos. En las Cuencas, ubicadas en la vertiente oriental de los Andes y en la Región Amazónica, se hicieron hallazgos importantes de petróleo y gas. Las Cuencas de Talara en el Norte, en la Cuenca Marañón en la selva peruana, a diciembre 2016, tienen una producción acumulada de 2,577 millones de barriles de petróleo.

En la Selva Sur, en la década de los años 1980, se descubrieron los yacimientos de Camisea, con reservas probadas de alrededor de 8,1 trillones de pies cúbicos y 567 millones de barriles de líquidos. Desde que entró en operaciones sumado a lo que salió de Talara ha producido 7,374 billones de pies cúbicos de gas (MINEM, 2017).

\section{Formaciones salinas, mantos de carbón no explotable y basaltos}

En estas cuencas sedimentarias también hay formaciones con salmueras, que no se han dimensionado a la fecha y que deben ser materia de estudio con fines del almacenamiento geológico de dióxido de carbono.

En el territorio peruano hay también depósitos importantes de rocas carbonáceas que son trabajadas a diferentes escalas, pero como ocurre en otros lugares del mundo hay mantos de carbón que no son de interés debido a sus características técnico-económicas y devienen en no explotables. Adicionalmente hay importante presencia de rocas volcánicas basálticas que deben también evaluarse respecto a su potencial de almacenamiento de $\mathrm{CO}_{2}$ (Figura 1).

Parte importante de las consideraciones es la investigación de los otros potenciales almacenes geológicos de dióxido de carbono, tales como los mantos de carbón no explotables, y las formaciones basálticas, que también constituyen ambientes favorables.

Existen en la Carta Geológica Nacional 211 regiones que están conformadas por basaltos, cuyas edades varían desde Cretácico Inferior (Ki-ch) hasta el Paleógeno y se distribuyen en 15 hojas de la carta y que deben ser caracterizadas con fines del almacenamiento geológico de $\mathrm{CO}_{2}$. El listado está en el Anexo 1.

\section{Metodología}

\section{Estimación de la capacidad de almacenamiento de los reservorios geológicos}

Los métodos disponibles para estimar volúmenes subsuperficiales se aplican amplia y rutinariamente en las estimaciones relacionadas con la eliminación de hidrocarburos, aguas subterráneas, almacenamiento subterráneo de gas natural y control de inyecciones subterráneas (Goodman et al., 2011). Normalmente se utilizan dos enfoques diferentes para estimar los volúmenes de inyección en el subsuelo; se definen como métodos estáticos y dinámicos (Calhoun, 1982). Los métodos estáticos utilizados para estimar el potencial de almacenamiento de $\mathrm{CO}_{2}$ se basan en modelos volumétricos y en modelos basados en la compresibilidad (Bachu et al., 2007; Burruss et al., 2009; Van der Meer y Yavuz, 2009). Los métodos volumétricos se aplican generalmente cuando se asume que la formación está abierta y que los fluidos de formación son desplazados de la formación o manejados vía producción. Si se demuestra que el sistema está cerrado, los volumétricos del sistema de almacenamiento seguirían calculándose, pero la estimación del recurso se basaría en la cantidad de ese espacio que podría comprimirse debido a la inyección de $\mathrm{CO}_{2}$. Normalmente, no se pueden realizar simulaciones dinámicas significativas antes de que se recojan datos específicos del campo de interés, tales como tasas de inyección medidas en campo y/o pruebas de pozos. La metodología US-DOE utiliza el enfoque volumétrico para estimar el potencial de recursos de almacenamiento de $\mathrm{CO}_{2}$ en yacimientos de petróleo y gas, formaciones salinas y vetas de carbón no minables, es la que será usada para este fin.

La metodología US-DOE se basa en métodos volumétricos para estimar volúmenes subsuperficiales, distribuciones de fluidos in situ y procesos de desplazamiento de fluidos (Calhoun, 1982).

Esta metodología es usada para evaluaciones de alto nivel de posibles yacimientos de almacenamiento de $\mathrm{CO}_{2}$ en Estados Unidos y Canadá; pero se ha convertido más o menos en estándar de facto en la comunidad académica internacional. Como ya se mencionó está 
basada en estimar el volumen de poros físicamente accesible. No incluye ninguna consideración de restricciones reguladoras o económicas, y es utilizado para políticas gubernamentales y decisiones comerciales de amplio alcance relacionadas con la energía.

Las estimaciones de recursos de almacenamiento de $\mathrm{CO}_{2}$, son fundamentalmente estimaciones del volumen de poro disponible de una formación dada que es accesible para $\mathrm{CO}_{2}$ inyectado a través de pozos perforados y completados, partiendo de la hipótesis de que los fluidos in situ serán desplazados por el $\mathrm{CO}_{2}$ inyectado a una distancia de la misma formación o formaciones vecinas o gestionados por medio de la producción, el tratamiento y la eliminación.

Las estimaciones del volumen de almacenamiento subterráneo dependen de las propiedades geológicas (área, espesor y porosidad de las formaciones) y la eficiencia del almacenamiento (la fracción del volumen de poro accesible que ocupará el $\mathrm{CO}_{2}$ inyectado).

La estimación en masa de recursos de almacenamiento de $\mathrm{CO}_{2}$ en yacimientos de petróleo y gas se obtiene de la siguiente ecuación:

$$
\mathrm{G}_{\mathrm{CO} 2}=\mathrm{Ah}_{\mathrm{n}} \Phi_{\mathrm{e}}\left(1-\mathrm{S}_{\mathrm{wi}}\right) \mathrm{B}_{\mathrm{C} 02 \mathrm{std}} \mathrm{E}_{\mathrm{oil} / \text { gas }}
$$

Donde A es el área que define el yacimiento de petróleo o gas que se está evaluando para el almacenamiento de $\mathrm{CO}_{2}, \mathrm{~h}_{\mathrm{n}}$ es la altura de la columna de petróleo y gas en el depósito, $\Phi_{\mathrm{e}}$ es la porosidad efectiva media en volumen definida por el espesor de la red, $\mathrm{S}_{\mathrm{wi}}$ es la saturación media inicial de agua dentro del área total (A) y espesor neto $\left(h_{n}\right), B$ es el factor de volumen de formación de fluido; $\rho \mathrm{CO}_{2 \text { std }}$ es la densidad estándar de $\mathrm{CO}_{2}$ evaluada a presión y temperatura estándar y $\mathrm{E}_{\text {oil/gas }}$ es el factor de eficiencia de almacenamiento de $\mathrm{CO}_{2}$; el factor $\mathrm{B}$, convierte el volumen estándar de aceite o gas en volumen subsuperficial (a presión y temperatura del depósito), por ejemplo, el volumen del tanque de almacenamiento de petróleo por volumen del yacimiento de petróleo, mientras que el factor de eficiencia de almacenamiento $\mathrm{E}_{\text {oilgas }}$ es el volumen de $\mathrm{CO}_{2}$ almacenado en un depósito de petróleo o gas por unidad de volumen de petróleo o gas original (OOIP o OGIP - Original Oil In Place u Original Gas In Place).

La estimación en masa de recursos de almacenamiento de $\mathrm{CO}_{2}$ en un acuífero salino profundo:

$$
\mathrm{G}_{\mathrm{CO2}}=\mathrm{A}_{\mathrm{t}} \mathrm{h}_{\mathrm{g}} \Phi_{\text {tot }} \rho \mathrm{E}_{\text {saline }}
$$

Donde $\mathrm{A}_{\mathrm{t}}$ es el área geográfica que deslinda la cuenca o región evaluada para el almacenamiento de $\mathrm{CO}_{2}, \mathrm{~h}_{\mathrm{g}}$ es el espesor bruto de las formaciones salinas para las que se evalúa el almacenamiento de $\mathrm{CO}_{2}$ en la cuenca o región de origen por $\mathrm{A}, \Phi_{\text {tot }}$ es la porosidad total en el volumen definido por el espesor neto, $\rho$ es la densidad de $\mathrm{CO}_{2}$ evaluada a presión y temperatura que representa las condiciones de almacenamiento previstas para una unidad geológica específica promediada sobre $\mathrm{h}_{\mathrm{g}} \mathrm{y} \mathrm{A}_{\mathrm{t}}$ y $\mathrm{E}_{\text {saline }}$ es el factor de eficiencia del almacenamiento de $\mathrm{CO}_{2}$ que retiene una fracción del volumen total de los poros que está lleno de $\mathrm{CO}_{2}$.

La estimación en masa de recursos de almacenamiento de $\mathrm{CO}_{2}$ de los mantos de carbón no minable.

$$
\mathrm{G}_{\mathrm{CO} 2}=\mathrm{Ah}_{\mathrm{g}} \mathrm{C}_{\mathrm{s}, \max } \rho_{\mathrm{CO} 2 \mathrm{std}} \mathrm{E}_{\text {coal }}
$$

Donde A es el área geográfica que delimita la cuenca o región carbonífera para el cálculo del almacenamiento de $\mathrm{CO}_{2}, \mathrm{~h}_{\mathrm{g}}$ es el espesor bruto de los mantos de carbón para las que se evalúa el almacenamiento de $\mathrm{CO}_{2}$ dentro de la cuenca o región determinada por A, $\mathrm{C}_{\mathrm{s}, \max }$ es el volumen estándar máximo adsorbido de $\mathrm{CO}_{2}$ por unidad de volumen de carbón in situ; asume condiciones de carbón saturado de $\mathrm{CO}_{2}$ al 100\%; si está libre de ceniza seca, se debe realizar la conversión, $\rho_{\mathrm{CO} 2 \text { std }}$ es la densidad estándar de $\mathrm{CO}_{2}, \mathrm{y} \mathrm{E}_{\text {coal }}$ es el factor de eficiencia de almacenamiento de $\mathrm{CO}_{2}$ que refleja una fracción del volumen total de carbón a granel que es contactado por el $\mathrm{CO}_{2}$.

Luego de la exploración, en la etapa de caracterización del sitio (ver Tabla 4), será de suma importancia determinar la inyectividad de cada formación geológica, entendiéndose que inyectividad es una medida cuantitativa de la facilidad con la que un gas o líquido o fluido en estado supercrítico, se introduce dentro de una formación geológica por unidad de espesor de la formación. Se deberá establecer también el diseño de la malla, la distribución espacial de los pozos de inyección y evaluar eventualmente la extracción de agua mientras se inyecta $\mathrm{CO}_{2}$.

Otro aspecto importante que no se puede soslayar en los tiempos actuales es la licencia social y el Perú como signatario de convenios internacionales, por ejemplo: El convenio 169 de la OIT (1989): pueblos indígenas e inclusión social, está en la obligación de consultar a los habitantes de las localidades y a los pueblos originarios aquellas decisiones que los puedan afectar directamente o aquellas que estén relacionadas con el uso de los recursos geológicos y/o del subsuelo en los 
territorios donde habitan. En esa línea los proyectos de captura y almacenamiento geológico de $\mathrm{CO}_{2}$, deberán de ser socializados desde etapas tempranas de su desarrollo de acuerdo con la normatividad existente.

\section{Conclusiones}

La captura y el secuestro o almacenamiento geológico de $\mathrm{CO}_{2}$ es la mejor alternativa para reducir el exceso de emisiones de $\mathrm{CO}_{2}$ antropogénico al medio ambiente, tanto por consideraciones técnicas como económicas. Los volúmenes de almacenamiento de $\mathrm{CO}_{2}$ y los tiempos de residencia prolongados en los depósitos geológicos lo hacen particularmente interesante.

El más importante potencial de secuestro o almacenamiento geológico de $\mathrm{CO}_{2}$ identificado en el Perú, está en los depósitos de hidrocarburos, tanto en los explotados y los que aún están en operación o por explotarse, y se emplaza en las cuencas de Talara y Marañón y Ucayali.

En el Perú, NO se han estudiado aún formaciones con acuíferos salinos profundos (entre 800 y 2500 $\mathrm{m}$ de profundidad), por lo tanto, hay mucho trabajo pendiente en esta área. Presumiblemente en el caso peruano éste debe ser el almacén geológico más importante que existe.

Las formaciones de volcánicos con basaltos tienen una amplia distribución en el territorio nacional y el trabajo que sigue es ubicarlos y precisar sus características para cuantificar su potencial de almacenamiento geológico de $\mathrm{CO}_{2}$.

\section{Agradecimientos}

Agradecemos a los revisores de éste artículo por sus importantes sugerencias para mejorarlo y darle el aspecto que finalmente tiene.

\section{Referencias}

Bachu, S. (2008). $\mathrm{CO}_{2}$ storage in geological media: Role, means, status and barriers to deployment. Progress in Energy and Combustion Science, $34(2), \quad 254-273$. https://doi.org/10.1016/j. pecs.2007.10.001
Bachu, S.; Bonijoly, D.; Bradshaw, J.; Burruss, R.; Holloway, S.; Christensen, N.P.; Mathiassen, O.M. (2007). $\mathrm{CO}_{2}$ storage capacity estimation: Methodology and gaps. International Journal of Greenhouse Gas Control, 1(4), 430-443. https:// doi.org/10.1016/S1750-5836(07)00086-2

Beltrán, L.; Dávila, M.; Contreras, C.; Arévalo, V.; Valenzuela, J.M.; Jiménez, O.; Medina, E. (2012). Atlas de Almacenamiento Geológico de $\mathrm{CO}_{2}$. Secretaria de Energía, Comisión Federal de Electricidad, México.

Bradshaw, B.E.; Spencer, L.K.; Lahtinen, A.L.; Khider, K.; Ryan, D.J.; Colwell, J.B.; Chirinos, A.; Bradshaw, J.; Draper, J.; Hodgkinson, J.; McKillop, M. (2011). An assessment of Queensland's $\mathrm{CO}_{2}$ geological storage prospectivity-The Queensland $\mathrm{CO}_{2}$ Geological Storage Atlas. Energy Procedia, 4, 4583-4590. https://doi.org/10.1016/j.egypro.2011.02.417

Burruss, R.C.; Brennan, S.T.; Freeman, P.A.; Merrill, M.D.; Ruppert, L.F.; Becker, M.F.; Herkelrath, W.N.; Kharaka, Y.K.; Neuzil, C.E.; Swanson, S.M.; Cook, T.A.; Klett, T.R.; Nelson, P.H.; Schenk, C.J. (2009). Development of a probabilistic assessment methodology for evaluation of carbon dioxide storage. U.S. Geological Survey OpenFile Report 2009-1035.

Calhoun, Jr., J.C. (1982). Fundamentals of reservoir engineering. Norman: University of Oklahoma Press.

Chadwick, A.; Arts, R.; Bernstone, C.; May, F.; Thibeau, S.; Zweigel, P. (2008). Best practice for the storage of $\mathrm{CO}_{2}$ in saline aquifers observations and guidelines from the SACS and CO2STORE projects. British Geological Survey.

ChemicaLogic Corporation (1999). Carbon Dioxide: Temperature - Pressure Diagram.

CO2CRC. (2012). The cooperative research centre for greenhouse gas technologies. What-is_CCS? CO2CRC. Consultado el 18 de octubre de 2018. http://www.co2crc.com.au/

Dirección General de Electricidad. (2016). Anuario ejecutivo de electricidad 2016. Ministerio de Energía y Minas, Lima, Perú. 
DOE - Laboratory, U.D. (2010). Carbon sequestration Atlas of the United States and Canada. U.S. Department of Energy's (DOE).

DOE \& NETL. (2015). Carbon Storage Atlas. (5th ed.). Department of Energy's (DOE) National Energy Technology Laboratory (NETL). http:// www.netl.doe.gov/

Frailey S.M.; Finley R.J. (2009). Classification of $\mathrm{CO}_{2}$ Geologic Storage: Resource and Capacity. Energy Procedia, 1(1), 2623-2630. https://doi. org/10.1016/j.egypro.2009.02.029

Goodman, A.; Hakala, A.; Bromhal, G.; Deel, D.; Rodosta, T.; Frailey, S.; Small, M.; Allen, D.; Romanov, V.; Fazio, J.; Huerta, N.; McIntyre, D.; Kutchko, B.; Guthrie, G. (2011). U.S. DOE methodology for the development of geologic storage potential for carbon dioxide at the national and regional scale. International Journal of Greenhouse Gas Control, 5(4), 952-965. https:// doi.org/10.1016/j.ijggc.2011.03.010

INGEMMET (2020). Mapa Geológico del Perú. INGEMMET, Perú.

Leung, D.Y.; Caramanna, G.; Maroto-Valer, M.M. (2014). An overview of current status of carbon dioxide capture and storage technologies. Renewable and Sustainable Energy Reviews, 39, 426-443. https://doi.org/10.1016/j. rser.2014.07.093

Medina-Ketzer, J.M.; Machado, C.X.; Rockett, G.C.; Iglesias, R.S. (2016). Atlas Brasileiro de
Captura e Armazenamento Geológico de $\mathrm{CO}_{2}$. Universitária da PUCRS.

MINEM (2017). Plan de desarrollo de los recursos de hidrocarburos 2017 - 2021. Ministerio de Energía y Minas.

Naciones Unidas UNFCC (2015). Aprobación del Acuerdo de Paris.

Norwegian Petroleum Directorate. (2014). $\mathrm{CO}_{2}$ Storage Atlas: Norwegian Continental Shelf. https://www. npd.no/en/facts/publications/co2-atlases/co2atlas-for-the-norwegian-continental-shelf/

OIT (1989). Convenio (N. 169) sobre pueblos indígenas y tribales en países independientes. C169. Organización Internacional del Trabajo.

PNUMA. (2005). La captación y el almacenamiento de dióxido de carbono. Grupo Intergubernamental de Expertos sobre el Cambio Climático.

Society of Petroleum Engineers. (2018). Sistema de Gerencia de los Recursos de Petróleo. Ed. Society of Petroleum Engineers and the Oil and Gas Reserves.

Van der Meer, L.G.H.; Yavuz, F. (2009). $\mathrm{CO}_{2}$ storage capacity calculations for the Dutch subsurface. Energy Procedia, 1(1), 2615-2622. https://doi. org/10.1016/j.egypro.2009.02.028

Trabajo recibido: abril 15 de 2019

Trabajo aceptado: agosto 31 de 2020 
Anexo 1. Formaciones con basaltos en la carta geológica nacional. Mapa geológico del Perú - 1:100 000 - 2018.

\begin{tabular}{|c|c|c|c|c|c|c|c|}
\hline Obj ID & Cod. & Descripción & Nombre & Unidad & Hoja & Área & Long \\
\hline 26249 & 2745 & Basalto & P-ba & Basalto & $32 \mathrm{u}$ & 0,000006 & 0,011018287 \\
\hline 26250 & 2745 & Basalto & P-ba & Basalto & $32 \mathrm{u}$ & 0,000002 & 0,0070631 \\
\hline 26251 & 2745 & Basalto & P-ba & Basalto & $32 \mathrm{u}$ & 0,000003 & 0,018197597 \\
\hline 26252 & 2745 & Basalto & P-ba & Basalto & $32 u$ & 0,000001 & 0,008914019 \\
\hline 26253 & 2745 & Basalto & P-ba & Basalto & $32 u$ & 0,000004 & 0,021040458 \\
\hline 26254 & 2745 & Basalto & P-ba & Basalto & $32 u$ & 0,000003 & 0,012963709 \\
\hline 26255 & 2745 & Basalto & P-ba & Basalto & $32 u$ & 0,000001 & 0,008897514 \\
\hline 26256 & 2745 & Basalto & P-ba & Basalto & $32 u$ & 0,000007 & 0,032304048 \\
\hline 89070 & 139 & $\begin{array}{l}\text { Basaltos gris oscuros de textura porfirítica con fenocristales de plagio- } \\
\text { clasas de olivino. }\end{array}$ & PN-cay & Formación Cayconi & $29 v$ & 0,000012 & 0,013459398 \\
\hline 89071 & 139 & $\begin{array}{l}\text { Basaltos gris oscuros de textura porfirítica con fenocristales de plagio- } \\
\text { clasas de olivino. }\end{array}$ & PN-cay & Formación Cayconi & $29 v$ & 0,000007 & 0,010919067 \\
\hline 89072 & 139 & $\begin{array}{l}\text { Basaltos gris oscuros de textura porfirítica con fenocristales de plagio- } \\
\text { clasas de olivino. }\end{array}$ & PN-cay & Formación Cayconi & $29 \mathrm{v}$ & 0,000020 & 0,021014726 \\
\hline 89073 & 139 & $\begin{array}{l}\text { Basaltos gris oscuros de textura porfirítica con fenocristales de plagio- } \\
\text { clasas de olivino. }\end{array}$ & PN-cay & Formación Cayconi & $29 v$ & 0,000141 & 0,056178127 \\
\hline 89120 & 139 & $\begin{array}{l}\text { Basaltos gris oscuros de textura porfirítica con fenocristales de plagio- } \\
\text { clasas de olivino. }\end{array}$ & PN-cay & Formación Cayconi & $29 v$ & 0,000105 & 0,060994189 \\
\hline 89121 & 139 & $\begin{array}{l}\text { Basaltos gris oscuros de textura porfirítica con fenocristales de plagio- } \\
\text { clasas de olivino. }\end{array}$ & PN-cay & Formación Cayconi & $29 v$ & 0,000024 & 0,022355905 \\
\hline 89245 & 139 & $\begin{array}{l}\text { Basaltos gris oscuros de textura porfirítica con fenocristales de plagio- } \\
\text { clasas de olivino. }\end{array}$ & PN-cay & Formación Cayconi & $29 \mathrm{v}$ & 0,000775 & 0,143682039 \\
\hline 89248 & 139 & $\begin{array}{l}\text { Basaltos gris oscuros de textura porfirítica con fenocristales de plagio- } \\
\text { clasas de olivino. }\end{array}$ & PN-cay & Formación Cayconi & $29 v$ & 0,000019 & 0,023557134 \\
\hline 89249 & 139 & $\begin{array}{l}\text { Basaltos gris oscuros de textura porfirítica con fenocristales de plagio- } \\
\text { clasas de olivino. }\end{array}$ & PN-cay & Formación Cayconi & $29 \mathrm{v}$ & 0,000063 & 0,054152709 \\
\hline 89250 & 139 & $\begin{array}{l}\text { Basaltos gris oscuros de textura porfirítica con fenocristales de plagio- } \\
\text { clasas de olivino. }\end{array}$ & PN-cay & Formación Cayconi & $29 v$ & 0,000056 & 0,043636955 \\
\hline 89254 & 139 & $\begin{array}{l}\text { Basaltos gris oscuros de textura porfirítica con fenocristales de plagio- } \\
\text { clasas de olivino. }\end{array}$ & PN-cay & Formación Cayconi & $29 \mathrm{v}$ & 0,000023 & 0,026339129 \\
\hline 89255 & 139 & $\begin{array}{l}\text { Basaltos gris oscuros de textura porfirítica con fenocristales de plagio- } \\
\text { clasas de olivino. }\end{array}$ & PN-cay & Formación Cayconi & $29 v$ & 0,000051 & 0,035781075 \\
\hline 89257 & 139 & $\begin{array}{l}\text { Basaltos gris oscuros de textura porfirítica con fenocristales de plagio- } \\
\text { clasas de olivino. }\end{array}$ & PN-cay & Formación Cayconi & $29 v$ & 0,000044 & 0,032132865 \\
\hline 108573 & 247 & Basaltos, areniscas y limoarcillas rojas. & Ki-ch & Formación Chayllacatana & 251 & 0,000024 & 0,065657993 \\
\hline
\end{tabular}




\begin{tabular}{|c|c|c|c|c|c|c|c|}
\hline Obj ID & Cod. & Descripción & Nombre & Unidad & Hoja & Área & Long \\
\hline 108689 & 247 & Basaltos, areniscas y limoarcillas rojas. & $\mathrm{Ki}$-ch & Formación Chayllacatana & 251 & 0,000020 & 0,046146509 \\
\hline 108690 & 247 & Basaltos, areniscas y limoarcillas rojas. & $\mathrm{Ki}$-ch & Formación Chayllacatana & 251 & 0,000055 & 0,139084036 \\
\hline 108696 & 247 & Basaltos, areniscas y limoarcillas rojas. & $\mathrm{Ki}$-ch & Formación Chayllacatana & 251 & 0,000039 & 0,09992425 \\
\hline 108741 & 247 & Basaltos, areniscas y limoarcillas rojas. & Ki-ch & Formación Chayllacatana & 251 & 0,000007 & 0,019604073 \\
\hline 108742 & 247 & Basaltos, areniscas y limoarcillas rojas. & $\mathrm{Ki}$-ch & Formación Chayllacatana & 251 & 0,000004 & 0,012938973 \\
\hline 108744 & 247 & Basaltos, areniscas y limoarcillas rojas. & Ki-ch & Formación Chayllacatana & 251 & 0,000063 & 0,165387388 \\
\hline 108865 & 247 & Basaltos, areniscas y limoarcillas rojas. & Ki-ch & Formación Chayllacatana & 251 & 0,000023 & 0,057279738 \\
\hline 108866 & 247 & Basaltos, areniscas y limoarcillas rojas. & $\mathrm{Ki}$-ch & Formación Chayllacatana & 251 & 0,000115 & 0,282496586 \\
\hline 108867 & 247 & Basaltos, areniscas y limoarcillas rojas. & Ki-ch & Formación Chayllacatana & 251 & 0,000113 & 0,280212212 \\
\hline 108872 & 247 & Basaltos, areniscas y limoarcillas rojas. & Ki-ch & Formación Chayllacatana & 251 & 0,000004 & 0,010274794 \\
\hline 108873 & 247 & Basaltos, areniscas y limoarcillas rojas. & Ki-ch & Formación Chayllacatana & 251 & 0,000011 & 0,02747867 \\
\hline 108874 & 247 & Basaltos, areniscas y limoarcillas rojas. & $\mathrm{Ki}$-ch & Formación Chayllacatana & 251 & 0,000073 & 0,18828744 \\
\hline 108875 & 247 & Basaltos, areniscas y limoarcillas rojas. & Ki-ch & Formación Chayllacatana & 251 & 0,000031 & 0,076052938 \\
\hline 108876 & 247 & Basaltos, areniscas y limoarcillas rojas. & $\mathrm{Ki}$-ch & Formación Chayllacatana & 251 & 0,000050 & 0,120818434 \\
\hline 108882 & 247 & Basaltos, areniscas y limoarcillas rojas. & $\mathrm{Ki}$-ch & Formación Chayllacatana & 251 & 0,000024 & 0,037315721 \\
\hline 108899 & 247 & Basaltos, areniscas y limoarcillas rojas. & Ki-ch & Formación Chayllacatana & 251 & 0,000005 & 0,014135769 \\
\hline 108900 & 247 & Basaltos, areniscas y limoarcillas rojas. & Ki-ch & Formación Chayllacatana & 251 & 0,000095 & 0,236545299 \\
\hline 108901 & 247 & Basaltos, areniscas y limoarcillas rojas. & Ki-ch & Formación Chayllacatana & 251 & 0,000039 & 0,099901028 \\
\hline 108902 & 247 & Basaltos, areniscas y limoarcillas rojas. & Ki-ch & Formación Chayllacatana & 251 & 0,000033 & 0,083040888 \\
\hline 109507 & 247 & Basaltos, areniscas y limoarcillas rojas. & $\mathrm{Ki}$-ch & Formación Chayllacatana & $25 \mathrm{~m}$ & 0,000000 & 0,002847194 \\
\hline 109509 & 247 & Basaltos, areniscas y limoarcillas rojas. & Ki-ch & Formación Chayllacatana & $25 \mathrm{~m}$ & 0,000028 & 0,067162545 \\
\hline 109808 & 247 & Basaltos, areniscas y limoarcillas rojas. & Ki-ch & Formación Chayllacatana & $25 \mathrm{~m}$ & 0,000002 & 0,006605013 \\
\hline 109839 & 247 & Basaltos, areniscas y limoarcillas rojas. & Ki-ch & Formación Chayllacatana & $25 \mathrm{~m}$ & 0,000046 & 0,103278533 \\
\hline 109840 & 247 & Basaltos, areniscas y limoarcillas rojas. & Ki-ch & Formación Chayllacatana & $25 \mathrm{~m}$ & 0,000003 & 0,009564841 \\
\hline 109841 & 247 & Basaltos, areniscas y limoarcillas rojas. & Ki-ch & Formación Chayllacatana & $25 \mathrm{~m}$ & 0,000008 & 0,023490345 \\
\hline 109842 & 247 & Basaltos, areniscas y limoarcillas rojas. & Ki-ch & Formación Chayllacatana & $25 \mathrm{~m}$ & 0,000039 & 0,074683909 \\
\hline 109870 & 247 & Basaltos, areniscas y limoarcillas rojas. & Ki-ch & Formación Chayllacatana & $25 \mathrm{~m}$ & 0,000047 & 0,106468027 \\
\hline 109930 & 247 & Basaltos, areniscas y limoarcillas rojas. & Ki-ch & Formación Chayllacatana & $25 \mathrm{~m}$ & 0,000023 & 0,065712174 \\
\hline 109931 & 247 & Basaltos, areniscas y limoarcillas rojas. & Ki-ch & Formación Chayllacatana & $25 \mathrm{~m}$ & 0,000006 & 0,017556514 \\
\hline
\end{tabular}


留 Continuación Anexo 1.

\begin{tabular}{|c|c|c|c|c|c|c|c|}
\hline Obj ID & Cod. & Descripción & Nombre & Unidad & Hoja & Área & Long \\
\hline 109932 & 247 & Basaltos, areniscas y limoarcillas rojas. & Ki-ch & Formación Chayllacatana & $25 \mathrm{~m}$ & 0,000008 & 0,018885631 \\
\hline 109933 & 247 & Basaltos, areniscas y limoarcillas rojas. & $\mathrm{Ki}$-ch & Formación Chayllacatana & $25 \mathrm{~m}$ & 0,000006 & 0,018385582 \\
\hline 109968 & 247 & Basaltos, areniscas y limoarcillas rojas. & Ki-ch & Formación Chayllacatana & $25 \mathrm{~m}$ & 0,000037 & 0,087328936 \\
\hline 109992 & 247 & Basaltos, areniscas y limoarcillas rojas. & Ki-ch & Formación Chayllacatana & $25 \mathrm{~m}$ & 0,000009 & 0,022485073 \\
\hline 109996 & 247 & Basaltos, areniscas y limoarcillas rojas. & Ki-ch & Formación Chayllacatana & $25 \mathrm{~m}$ & 0,000011 & 0,027455023 \\
\hline 109997 & 247 & Basaltos, areniscas y limoarcillas rojas. & Ki-ch & Formación Chayllacatana & $25 \mathrm{~m}$ & 0,000010 & 0,024397227 \\
\hline 109998 & 247 & Basaltos, areniscas y limoarcillas rojas. & $\mathrm{Ki}-\mathrm{ch}$ & Formación Chayllacatana & $25 \mathrm{~m}$ & 0,000004 & 0,011743396 \\
\hline 110018 & 247 & Basaltos, areniscas y limoarcillas rojas. & Ki-ch & Formación Chayllacatana & $25 \mathrm{~m}$ & 0,000003 & 0,008206609 \\
\hline 110019 & 247 & Basaltos, areniscas y limoarcillas rojas. & Ki-ch & Formación Chayllacatana & $25 \mathrm{~m}$ & 0,000005 & 0,013172885 \\
\hline 110020 & 247 & Basaltos, areniscas y limoarcillas rojas. & Ki-ch & Formación Chayllacatana & $25 \mathrm{~m}$ & 0,000003 & 0,010611795 \\
\hline 110021 & 247 & Basaltos, areniscas y limoarcillas rojas. & Ki-ch & Formación Chayllacatana & $25 \mathrm{~m}$ & 0,000018 & 0,041272425 \\
\hline 110022 & 247 & Basaltos, areniscas y limoarcillas rojas. & $\mathrm{Ki}-\mathrm{ch}$ & Formación Chayllacatana & $25 \mathrm{~m}$ & 0,000049 & 0,110907976 \\
\hline 110024 & 247 & Basaltos, areniscas y limoarcillas rojas. & Ki-ch & Formación Chayllacatana & $25 \mathrm{~m}$ & 0,000013 & 0,030694194 \\
\hline 110025 & 247 & Basaltos, areniscas y limoarcillas rojas. & Ki-ch & Formación Chayllacatana & $25 \mathrm{~m}$ & 0,000025 & 0,058802505 \\
\hline 110026 & 247 & Basaltos, areniscas y limoarcillas rojas. & Ki-ch & Formación Chayllacatana & $25 \mathrm{~m}$ & 0,000043 & 0,099804026 \\
\hline 110027 & 247 & Basaltos, areniscas y limoarcillas rojas. & Ki-ch & Formación Chayllacatana & $25 \mathrm{~m}$ & 0,000038 & 0,085681664 \\
\hline 110028 & 247 & Basaltos, areniscas y limoarcillas rojas. & Ki-ch & Formación Chayllacatana & $25 \mathrm{~m}$ & 0,000021 & 0,049861811 \\
\hline 110029 & 247 & Basaltos, areniscas y limoarcillas rojas. & Ki-ch & Formación Chayllacatana & $25 \mathrm{~m}$ & 0,000051 & 0,116846795 \\
\hline 114648 & 247 & Basaltos, areniscas y limoarcillas rojas. & Ki-ch & Formación Chayllacatana & $26 \mathrm{~m}$ & 0,000114 & 0,268703711 \\
\hline 114653 & 247 & Basaltos, areniscas y limoarcillas rojas. & Ki-ch & Formación Chayllacatana & $26 \mathrm{~m}$ & 0,000039 & 0,119802399 \\
\hline 114654 & 247 & Basaltos, areniscas y limoarcillas rojas. & $\mathrm{Ki}-\mathrm{ch}$ & Formación Chayllacatana & $26 \mathrm{~m}$ & 0,000004 & 0,015035166 \\
\hline 114655 & 247 & Basaltos, areniscas y limoarcillas rojas. & Ki-ch & Formación Chayllacatana & $26 \mathrm{~m}$ & 0,000008 & 0,028597103 \\
\hline 114656 & 247 & Basaltos, areniscas y limoarcillas rojas. & $\mathrm{Ki}-\mathrm{ch}$ & Formación Chayllacatana & $26 \mathrm{~m}$ & 0,000149 & 0,32068859 \\
\hline 114660 & 247 & Basaltos, areniscas y limoarcillas rojas. & Ki-ch & Formación Chayllacatana & $26 \mathrm{~m}$ & 0,000033 & 0,10173469 \\
\hline 114927 & 247 & Basaltos, areniscas y limoarcillas rojas. & Ki-ch & Formación Chayllacatana & $26 \mathrm{~m}$ & 0,000001 & 0,004277999 \\
\hline 115129 & 247 & Basaltos, areniscas y limoarcillas rojas. & Ki-ch & Formación Chayllacatana & $26 n$ & 0,000012 & 0,028022956 \\
\hline 115130 & 247 & Basaltos, areniscas y limoarcillas rojas. & Ki-ch & Formación Chayllacatana & $26 n$ & 0,000030 & 0,036140598 \\
\hline 115152 & 247 & Basaltos, areniscas y limoarcillas rojas. & Ki-ch & Formación Chayllacatana & $26 n$ & 0,000002 & 0,006600665 \\
\hline 115153 & 247 & Basaltos, areniscas y limoarcillas rojas. & $\mathrm{Ki}-\mathrm{ch}$ & Formación Chayllacatana & $26 n$ & 0,000378 & 0,230501314 \\
\hline
\end{tabular}




\begin{tabular}{|c|c|c|c|c|c|c|c|}
\hline Obj ID & Cod. & Descripción & Nombre & Unidad & Hoja & Área & Long \\
\hline 115154 & 247 & Basaltos, areniscas y limoarcillas rojas. & Ki-ch & Formación Chayllacatana & $26 n$ & 0,000032 & 0,055921616 \\
\hline 115155 & 247 & Basaltos, areniscas y limoarcillas rojas. & Ki-ch & Formación Chayllacatana & $26 n$ & 0,000016 & 0,023238396 \\
\hline 115156 & 247 & Basaltos, areniscas y limoarcillas rojas. & Ki-ch & Formación Chayllacatana & $26 n$ & 0,000008 & 0,015923746 \\
\hline 115257 & 247 & Basaltos, areniscas y limoarcillas rojas. & $\mathrm{Ki}$-ch & Formación Chayllacatana & $26 n$ & 0,000005 & 0,012821568 \\
\hline 115258 & 247 & Basaltos, areniscas y limoarcillas rojas. & Ki-ch & Formación Chayllacatana & $26 n$ & 0,000037 & 0,053347879 \\
\hline 115259 & 247 & Basaltos, areniscas y limoarcillas rojas. & Ki-ch & Formación Chayllacatana & $26 n$ & 0,000060 & 0,076790022 \\
\hline 115302 & 247 & Basaltos, areniscas y limoarcillas rojas. & $\mathrm{Ki}-\mathrm{ch}$ & Formación Chayllacatana & $26 n$ & 0,000027 & 0,045071037 \\
\hline 115322 & 247 & Basaltos, areniscas y limoarcillas rojas. & Ki-ch & Formación Chayllacatana & $26 n$ & 0,000051 & 0,081527404 \\
\hline 115323 & 247 & Basaltos, areniscas y limoarcillas rojas. & Ki-ch & Formación Chayllacatana & $26 n$ & 0,000099 & 0,085470574 \\
\hline 115324 & 247 & Basaltos, areniscas y limoarcillas rojas. & Ki-ch & Formación Chayllacatana & $26 n$ & 0,000007 & 0,012243766 \\
\hline 115325 & 247 & Basaltos, areniscas y limoarcillas rojas. & $\mathrm{Ki}$-ch & Formación Chayllacatana & $26 n$ & 0,000154 & 0,118633423 \\
\hline 115326 & 247 & Basaltos, areniscas y limoarcillas rojas. & $\mathrm{Ki}$-ch & Formación Chayllacatana & $26 n$ & 0,000035 & 0,062430567 \\
\hline 115327 & 247 & Basaltos, areniscas y limoarcillas rojas. & Ki-ch & Formación Chayllacatana & $26 n$ & 0,000038 & 0,056656844 \\
\hline 115328 & 247 & Basaltos, areniscas y limoarcillas rojas. & $\mathrm{Ki}$-ch & Formación Chayllacatana & $26 n$ & 0,000031 & 0,029645507 \\
\hline 115341 & 247 & Basaltos, areniscas y limoarcillas rojas. & $\mathrm{Ki}-\mathrm{ch}$ & Formación Chayllacatana & $26 n$ & 0,000006 & 0,017711305 \\
\hline 115342 & 247 & Basaltos, areniscas y limoarcillas rojas. & $\mathrm{Ki}-\mathrm{ch}$ & Formación Chayllacatana & $26 n$ & 0,000046 & 0,046484914 \\
\hline 115344 & 247 & Basaltos, areniscas y limoarcillas rojas. & Ki-ch & Formación Chayllacatana & $26 n$ & 0,000120 & 0,114733667 \\
\hline 115345 & 247 & Basaltos, areniscas y limoarcillas rojas. & Ki-ch & Formación Chayllacatana & $26 n$ & 0,000090 & 0,120392569 \\
\hline 115350 & 247 & Basaltos, areniscas y limoarcillas rojas. & $\mathrm{Ki}$-ch & Formación Chayllacatana & $26 n$ & 0,000037 & 0,04524267 \\
\hline 115366 & 247 & Basaltos, areniscas y limoarcillas rojas. & $\mathrm{Ki}$-ch & Formación Chayllacatana & $26 n$ & 0,000120 & 0,117886639 \\
\hline 115367 & 247 & Basaltos, areniscas y limoarcillas rojas. & Ki-ch & Formación Chayllacatana & $26 n$ & 0,000032 & 0,067464939 \\
\hline 115368 & 247 & Basaltos, areniscas y limoarcillas rojas. & Ki-ch & Formación Chayllacatana & $26 n$ & 0,000018 & 0,033390239 \\
\hline 115442 & 247 & Basaltos, areniscas y limoarcillas rojas. & Ki-ch & Formación Chayllacatana & $26 n$ & 0,000153 & 0,105659774 \\
\hline 115449 & 247 & Basaltos, areniscas y limoarcillas rojas. & Ki-ch & Formación Chayllacatana & $26 n$ & 0,000064 & 0,124623022 \\
\hline 115450 & 247 & Basaltos, areniscas y limoarcillas rojas. & Ki-ch & Formación Chayllacatana & $26 n$ & 0,000079 & 0,086580385 \\
\hline 115451 & 247 & Basaltos, areniscas y limoarcillas rojas. & Ki-ch & Formación Chayllacatana & $26 n$ & 0,000151 & 0,180010371 \\
\hline 115452 & 247 & Basaltos, areniscas y limoarcillas rojas. & $\mathrm{Ki}$-ch & Formación Chayllacatana & $26 n$ & 0,000056 & 0,045351984 \\
\hline 115453 & 247 & Basaltos, areniscas y limoarcillas rojas. & Ki-ch & Formación Chayllacatana & $26 n$ & 0,000006 & 0,013249368 \\
\hline 115454 & 247 & Basaltos, areniscas y limoarcillas rojas. & $\mathrm{Ki}$-ch & Formación Chayllacatana & $26 n$ & 0,000063 & 0,062139602 \\
\hline
\end{tabular}


气 Continuación Anexo 1.

\begin{tabular}{|c|c|c|c|c|c|c|c|}
\hline Obj ID & Cod. & Descripción & Nombre & Unidad & Hoja & Área & Long \\
\hline 115455 & 247 & Basaltos, areniscas y limoarcillas rojas. & Ki-ch & Formación Chayllacatana & $26 n$ & 0,000206 & 0,130582522 \\
\hline 115539 & 1282 & Coladas volcánicas de basalto. & Nm-ru/i & $\begin{array}{l}\text { Formación Rumichaca - Miembro } \\
\text { inferior }\end{array}$ & $26 n$ & 0,000113 & 0,089185605 \\
\hline 115839 & 247 & Basaltos, areniscas y limoarcillas rojas. & Ki-ch & Formación Chayllacatana & $26 n$ & 0,000236 & 0,278233207 \\
\hline 119823 & 247 & Basaltos, areniscas y limoarcillas rojas. & $\mathrm{Ki}-\mathrm{ch}$ & Formación Chayllacatana & $27 \mathrm{n}$ & 0,000042 & 0,049407291 \\
\hline 119854 & 247 & Basaltos, areniscas y limoarcillas rojas. & $\mathrm{Ki}-\mathrm{ch}$ & Formación Chayllacatana & $27 \mathrm{n}$ & 0,000019 & 0,036975766 \\
\hline 119855 & 247 & Basaltos, areniscas y limoarcillas rojas. & Ki-ch & Formación Chayllacatana & $27 \mathrm{n}$ & 0,000026 & 0,03076005 \\
\hline 141202 & 247 & Basaltos, areniscas y limoarcillas rojas. & $\mathrm{Ki}-\mathrm{ch}$ & Formación Chayllacatana & $22 \mathrm{k}$ & 0,000035 & 0,061775297 \\
\hline 144738 & 247 & Basaltos, areniscas y limoarcillas rojas. & Ki-ch & Formación Chayllacatana & $23 \mathrm{k}$ & 0,000015 & 0,034314198 \\
\hline 144739 & 247 & Basaltos, areniscas y limoarcillas rojas. & $\mathrm{Ki}-\mathrm{ch}$ & Formación Chayllacatana & $23 \mathrm{k}$ & 0,000025 & 0,050840851 \\
\hline 144740 & 247 & Basaltos, areniscas y limoarcillas rojas. & $\mathrm{Ki}-\mathrm{ch}$ & Formación Chayllacatana & $23 \mathrm{k}$ & 0,000007 & 0,022907407 \\
\hline 144741 & 247 & Basaltos, areniscas y limoarcillas rojas. & Ki-ch & Formación Chayllacatana & $23 \mathrm{k}$ & 0,000022 & 0,050371252 \\
\hline 144742 & 247 & Basaltos, areniscas y limoarcillas rojas. & $\mathrm{Ki}$-ch & Formación Chayllacatana & $23 \mathrm{k}$ & 0,000032 & 0,051396973 \\
\hline 144743 & 247 & Basaltos, areniscas y limoarcillas rojas. & Ki-ch & Formación Chayllacatana & $23 \mathrm{k}$ & 0,000017 & 0,035839153 \\
\hline 144744 & 247 & Basaltos, areniscas y limoarcillas rojas. & Ki-ch & Formación Chayllacatana & $23 \mathrm{k}$ & 0,000046 & 0,069384417 \\
\hline 145010 & 247 & Basaltos, areniscas y limoarcillas rojas. & $\mathrm{Ki}$-ch & Formación Chayllacatana & $23 \mathrm{k}$ & 0,000027 & 0,041177514 \\
\hline 145012 & 247 & Basaltos, areniscas y limoarcillas rojas. & Ki-ch & Formación Chayllacatana & $23 \mathrm{k}$ & 0,000007 & 0,014437254 \\
\hline 148610 & 5230 & Basalto traquiandesita basaltica & N-ba,taba & Sills o coladas de basalto (N-B) & $24 \mathrm{k}$ & 0,000001 & 0,006340751 \\
\hline 149450 & 5230 & Basalto traquiandesita basaltica & N-ba,taba & Sills o coladas de basalto (N-B) & 241 & 0,000074 & 0,075068459 \\
\hline 149453 & 5230 & Basalto traquiandesita basaltica & N-ba,taba & Sills o coladas de basalto (N-B) & 241 & 0,000139 & 0,135172841 \\
\hline 149454 & 5230 & Basalto traquiandesita basaltica & N-ba,taba & Sills o coladas de basalto (N-B) & 241 & 0,000112 & 0,188466847 \\
\hline 149455 & 5230 & Basalto traquiandesita basaltica & N-ba,taba & Sills o coladas de basalto (N-B) & 241 & 0,000004 & 0,012110927 \\
\hline 149456 & 5230 & Basalto traquiandesita basaltica & N-ba,taba & Sills o coladas de basalto (N-B) & 241 & 0,000050 & 0,068132825 \\
\hline 149459 & 5230 & Basalto traquiandesita basaltica & N-ba,taba & Sills o coladas de basalto (N-B) & 241 & 0,000030 & 0,061258682 \\
\hline 149460 & 5230 & Basalto traquiandesita basaltica & N-ba,taba & Sills o coladas de basalto (N-B) & 241 & 0,000275 & 0,261132053 \\
\hline 149461 & 5230 & Basalto traquiandesita basaltica & N-ba,taba & Sills o coladas de basalto (N-B) & 241 & 0,000140 & 0,168754677 \\
\hline
\end{tabular}

